\title{
Los factores personales e institucionales en el sentido de pertenencia de estudiantes chilenos a lo largo de sus estudios superiores
}

Teresa Pino-Vera, Eduardo Cavieres-Fernández y José-Antonio Muñoz-Reyes

\section{RESUMEN}

El sentido de pertenencia es considerado en la literatura como un aspecto fundamental para desarrollar exitosamente los estudios superiores. El objetivo de este trabajo es entender la relación que existe entre el sentido de pertenencia y diversas variables personales (motivación) e institucionales (integración social y clima institucional) con las que está asociado. También se busca explorar las diferencias en esta relación a lo largo de los años de estudio. Para ello, la presente investigación explora el sentido de pertenencia de 459 estudiantes, en distintos años de su formación y pertenecientes a una unidad académica de una universidad chilena. A la luz de los resultados, se discute sus implicaciones para la promoción del sentido de pertenencia en las instituciones de educación superior.

Palabras clave: estudiantes, sentido de pertenencia, éxito en los estudios, educación superior, Chile.

Teresa Pino-Vera

Chilena. Doctora en Políticas y Gestión Educativa de la Universidad de Playa Ancha, Chile. Jefa de Carrera de la Universidad de Valparaíso, Chile. Temas de investigación: deserción en la enseñanza superior y el pre-requisito en el avance curricular.

Eduardo Cavieres-Fernández

Chileno. Doctor en Educación, Universidad de Wisconsin-Madison, EUA. Investigador del Centro de Estudios Avanzados, Universidad de Playa Ancha, Chile. Temas de investigación: currículo escolar y en educación superior. cooperación, etcétera). 


\title{
Os fatores pessoais e institucionais no sentido de pertença de estudantes chilenos ao longo de seus estudos superiores
}

\section{RESUMO}

O sentido de pertença é considerado na literatura como um aspecto fundamental para desenvolver de forma exitosa os estudos superiores. O objetivo deste trabalho é entender a relação que existe entre o sentido de pertença e diversas variáveis pessoais (motivação) e institucionais (integração social e clima institucional) com as que está associado. Também se busca explorar as diferenças nesta relação ao longo dos anos de estudo. Para isso, a presente pesquisa explora o sentido de pertença de 459 estudantes, em diferentes anos de sua formação e pertencentes a uma unidade acadêmica de uma universidade chilena. A luz dos resultados, se discute suas implicações para a promoção do sentido de pertença nas instituições de educação superior.

Palavras chave: estudantes, sentido de pertença, êxito nos estudos, educação superior, Chile.

\section{The personal and institutional factors that typify the sense of belonging of Chilean students throughout their higher education}

\begin{abstract}
The sense of belonging is considered in the literature as a fundamental aspect to successfully develop higher education. The objective of this paper is to understand the relationship that exists between the sense of belonging and diverse variables with which it is associated, either personal (such as motivation) or institutional (such as social integration and institutional climate). It also seeks to explore the differences in this relationship over the years of study. To do so, the present investigation explores the sense of belonging of 459 students who belong to an academic unit of a Chilean university and are enrolled in different years of their degree. Finally, its implications for the promotion of the sense of belonging in higher education institutions are discussed in light of the results.
\end{abstract}

Key words: students, sense of belonging, success in studies, higher education, Chile.

Recepción: 25/01/16. Aprobación: 07/04/17. 


\section{Introducción}

La permanencia exitosa de los estudiantes a lo largo de sus estudios universitarios ha adquirido gran importancia por las consecuencias positivas que trae para los propios estudiantes, sus familias y las instituciones de educación superior (Brunner, 2013; Himmel, 2002; Tinto, 2012). Con relación a ello, dentro de la literatura anglosajona diversos modelos teóricos han procurado explicar la permanencia o deserción de los estudiantes en sus estudios superiores, señalando los factores que estarían influyendo. ${ }^{1}$ Aunque hay diferencias entre estos modelos, en general coinciden en un vasto espectro de variables que incluyen aspectos personales de los estudiantes (es decir, antecedentes familiares, disposiciones motivacionales), así como variables institucionales referidas a la integración social (tales como relaciones con pares y profesores) y al clima institucional de las universidades (relación entre la sociabilidad de los estudiantes y la cultura interna de la institución). En ese contexto, el sentido de pertenencia emerge como un factor vinculado con aspectos personales e institucionales y, por tanto, permite una mirada integrada respecto a las variables que inciden en que los estudiantes desarrollen exitosamente sus estudios (Hurtado y Carter, 1997).

En América Latina, múltiples trabajos integran las diversas perspectivas y variables aportadas por estos modelos acerca de los factores que inciden en los estudiantes al desarrollar sus estudios superiores (Castaño, Gallón, Gómez y Vásquez, 2006; Osorio, Bolancé y Castillo-Caicedo, 2012; De Vries, León, Romero y Hernández, 2011; Donoso y Schiefelbein, 2007). En Chile, aunque aún escasos, los estudios que consideran estos factores confirman su importancia para que los estudiantes decidan avanzar o no en sus estudios (Saldaña y Barriga, 2010; Díaz, 2008; Abello, Diaz, Pérez, Almeida, Lagos, González y Strickland, 2012; Canales y De los Ríos, 2009). No obstante, el sentido de pertenencia ha recibido sólo una mención tangencial, aunque reconociendo su relevancia para los estudiantes (Abello et al., 2012).

El presente estudio, por tanto, explora la variable del sentido de pertenencia y su asociación con distintos factores personales e institucionales en 459 estudiantes, los que se encuentran en distintos años de su formación y pertenecen a una unidad académica de una universidad regional pública chilena. Específicamente, su objetivo es entender la relación que existe entre el sentido de pertenencia de estos estudiantes chilenos y distintos factores personales (motivación) e institucionales (integración social y clima institucional). También se busca explorar las diferencias en esta relación a lo largo de sus años de estudio. A la luz de estos resultados, se discuten sus implicaciones en la promoción del sentido de pertenencia en las instituciones de educación superior dada su importancia para que los estudiantes desarrollen exitosamente sus estudios.

\section{Sentido de pertenencia}

Desde la sociología, el estudio de Bollen y Hoyle (1990) define el sentido de pertenencia como la percepción del individuo respecto de cuánto se siente "pegado" o formando parte de un grupo. Esta definición se relaciona con el concepto de integración o ajuste por parte de los estudiantes al clima institucional, elemento central en los modelos de abandono y permanencia de los estudiantes en la educación superior (Tinto, 1993; Eaton y Bean, 1995; Nora y Cabrera, 1996). Por ende, en el contexto de dichos modelos, el sentido de pertenencia se refiere a la

\footnotetext{
${ }^{1}$ Si bien este artículo no trata sobre los modelos en sí mismos, se sustenta en sus aportes. Estos modelos han surgido a partir de modelos iniciales, que autores posteriores han ido enriqueciendo. Desde esa perspectiva, forman parte del mismo campo de estudio. Entre estos modelos destaca el de Deserción, enfocado en el estudiante de primer año, su capital cultural, antecedentes escolares, etcétera. (Spady, 1971; Tinto, 1975; Bean y Metzer, 1985; Ethington, 1990). Le han seguido el Modelo de Adaptación y Permanencia que incluye el factor motivación proveniente desde los padres (Nora y Cabrera, 1996) y el Modelo del Compromiso del Alumno (Nora, 2004) que incorpora factores que afectan la responsabilidad asumida por éste y su compromiso con los estudios.
} 
experiencia subjetiva, afectiva y cognitiva del estudiante de saberse formando parte e identificándose con la institución en donde desarrolla sus estudios superiores (Hurtado y Carter, 1997). Aquello incluye el sentirse un miembro valioso dentro de la institución universitaria y apreciar el ser parte de ella con los valores y normas que la constituyen (Haussmann, Schofield y Woods, 2007; Wolf-Wendel, Ward y Kinzie, 2009). Consecuentemente, el sentido de pertenencia, y por tanto la afinidad de los estudiantes con la institución educativa, constituye una variable que influye en su intención de permanecer o abandonar sus estudios (Kember, Lee y Li, 2001).

Desde esa perspectiva, un aspecto central del sentido de pertenencia se refiere a las relaciones positivas que los estudiantes establecen con sus pares y profesores y que les hace sentirse seguros y apoyados dentro de la institución, lo que incide positivamente en sus logros académicos (Kember y Leung, 2004; Johnson, Soldner, Leonard, Alvarez, Inkelas, RowanKenyon y Longerbeam, 2007). Al respecto, O'Keeffe (2013) ha señalado la importancia de que las instituciones de estudios superiores fomenten las buenas relaciones entre estudiantes y profesores puesto que están directa y fuertemente correlacionadas con la satisfacción de los estudiantes por sus estudios y su intención de permanecer en ellos, y viceversa (una mala relación podría estar directamente relacionada con su intención de abandonar los estudios). Por su parte, Hoffman, Richmond, Morrow y Salomone (2002/2003) indican que el sentido de pertenencia está influido por la habilidad de los estudiantes para establecer relaciones de apoyo entre ellos, ante los desafios propios de los estudios superiores.

Junto a lo anterior, el carácter subjetivo del sentido de pertenencia ha servido para cuestionar una visión que busca promover la inserción de los estudiantes en los centros educativos, sólo a partir de la asimilación que hagan éstos de las reglas y prácticas institucionales, sin que se valoren, además, los recursos y las experiencias culturales que traen desde sus contextos de origen (Hurtado y Carter, 1997). De este modo, diversas investigaciones sobre sentido de pertenencia han resaltado la necesidad de que las instituciones universitarias consideren la diversidad que traen los estudiantes, incorporándola a la cultura institucional para promover la pertenencia y, por tanto, la permanencia de estos estudiantes en sus estudios (Read, Archer y Leathwood, 2003; Maestas, Vaquera y Muñoz, 2007). Así, mientras Strayhon (2008) demuestra empíricamente la importancia de que los estudiantes compartan con grupos que pertenecen a su mismo ambiente cultural de origen ayudándoles a sentirse más integrados a la institución; Ostrove y Long (2007) muestran que la falta de consideración, por parte de las universidades, de aquellos aspectos objetivos y subjetivos que caracterizan a los grupos de clases sociales menos aventajadas, influye en su menor sentido de pertenencia y, por tanto, en su menor éxito académico.

Consecuentemente, diversos estudios se han focalizado en el sentido de pertenencia como factor de permanencia y han justificado su consistencia interna como variable diferenciable de otras que influyen en la formación académica de los estudiantes (Hoffman et al., 2002/2003; Haussmann et al., 2007; Nora y Cabrera, 1993). No obstante, la presente investigación reconoce la asociación establecida en la literatura entre esta variable y otras de carácter personal e institucional que inciden en la permanencia de los estudiantes a lo largo de sus estudios. De este modo, en esta investigación el sentido de pertenencia se analiza en relación con las siguientes variables: motivación (personal), integración social (variable institucional) y clima institucional (variable institucional). Asimismo, puesto que algunas investigaciones advierten que la influencia de estas variables sobre el sentido de pertenencia de los estudiantes varía en el tiempo (Hurtado y Carter, 1997; Haussmann et al., 2007), se considera igualmente este aspecto. En consecuencia, mientras diversas investigaciones se focalizan en estudiantes de primer año de estudios 
superiores, en este trabajo se consideran estudiantes que se encuentran en distintas etapas de su carrera. A continuación, se exponen las variables asociadas al sentido de pertenencia que son contempladas en este estudio.

\section{Integración social}

La integración social es el factor que más se ha asociado al sentido de pertenencia; incluso se podría considerar como parte del mismo fenómeno, pero mirado desde distintas perspectivas. De acuerdo con Hurtado y Carter (1997), mientras que el sentido de pertenencia se focaliza en la experiencia subjetiva de los estudiantes, la integración social se centra en aquellos comportamientos de los estudiantes que son considerados relevantes por las instituciones universitarias. Wolf-Wendel, Ward y Kinzie (2009) precisan que la integración social se refiere al grado con que los estudiantes llegan a compartir el sistema de creencias y comportamientos de sus compañeros y profesores, y se adhieren a las normas y requerimientos de la institución. Por tanto, la integración social hace hincapié en los tipos de interacción, tanto a través de actividades curriculares como extra-curriculares, que los estudiantes establecen con otros miembros de la universidad, ayudándoles a progresar y a sentirse parte de ella (Tinto, 1993; 2006/2007; Wilcox, Winn y Fyvie-Gauld, 2005). Desde esa perspectiva, los niveles de participación de los estudiantes dentro de la universidad constituyen una medida de integración social que se asocia positivamente con su sentido de pertenencia (Maestas, Vaquera y Muñoz, 2007).

Zepke, Leach y Prebble (2006) han planteado que la integración social no sólo debe considerar el esfuerzo de los estudiantes por adaptarse a una realidad institucional, sino que debe incluir las iniciativas que las instituciones llevan a cabo para adaptarse a su vez a las necesidades de los estudiantes, haciéndoles sentir parte de ellas. Así, Braxton y McClendon (2001/2002) mencionan diversas actividades a ser implementadas en los centros de educación superior, tales como la mentoría, la creación de canales comunicacionales efectivos para informar a los estudiantes y la promoción de prácticas pedagógicas activas por parte de los profesores que integren a los estudiantes por medio de actividades participativas y colaborativas. Igualmente, Wolf-Wendel, Ward y Kinzie (2009) han señalado la cercanía del concepto de integración social con el de involucramiento. Para Kuh, Cruce, Shoup, Kinzie y Gonyea (2008), el involucramiento representa el tiempo y la energía que los estudiantes ocupan en actividades educacionales que consideran importantes y que son ofrecidas por las instituciones a las que pertenecen. Hu (2011) precisa que estas actividades, tanto dentro como fuera de la sala de clases, se refieren fundamentalmente a aquellas que contribuyen al aprendizaje de los estudiantes y a su desarrollo personal.

\section{El clima institucional}

El clima institucional es otra variable que se asocia positivamente con el sentido de pertenencia, redundando en los logros académicos de los estudiantes (Strayhon, 2008; Meeuwise, Severiens y Born, 2010). Los estudios plantean que un clima institucional que acoge y apoya las diferencias y necesidades de los estudiantes de educación superior incide en que éstos se sientan parte de la institución (Hurtado y Carter, 1997; Locks, Hurtado, Bowman y Oseguera, 2008). Este clima institucional se caracteriza por estar abierto a que los grupos interactúen y establezcan relaciones de apoyo (Hurtado y Ponjuan, 2005). Por tanto, las relaciones que los estudiantes establecen se definen por el respeto, la amistad y la confianza (Johnson et al., 2007). Desde esa perspectiva, conforme a Nuñez (2009), la relación entre clima institucional y sentido de pertenencia contiene una dimensión psicológica (que los estudiantes perciban el ambiente como acogedor) y comportamental (que efectivamente se establezcan relaciones entre estudiantes y entre éstos con los profesores u otros miembros de la institución). 


\section{Motivación}

Son escasos los estudios que se adentran en la relación entre sentido de pertenencia y la motivación de los estudiantes hacia sus estudios, posiblemente por la vinculación de la pertenencia a modelos que subrayan más la integración social y el clima institucional (Neuville, Frenay, Schmitz, Boudrenghien, Noël y Wertz, 2007). No obstante, en la medida que esta variable incluye aspectos subjetivos de los estudiantes, justifica ser asociada a modelos que consideran variables más personales. Dentro de las pocas excepciones, se encuentran las investigaciones con estudiantes de enseñanza secundaria en EUA. Así, Goodenow (1993) y Anderman (2003) reportan la asociación positiva entre sentido de pertenencia y variables motivacionales tales como las expectativas de los estudiantes por sus logros académicos y la valoración por sus actividades de aprendizaje. Construyendo sobre estos resultados, Freeman, Anderman y Jensen (2007), en su investigación con estudiantes universitarios, concluyen que el sentido de pertenencia de éstos a una asignatura de la carrera en la que estudian, se asocia significativamente a tres factores motivacionales: la valoración por las tareas académicas (la percepción de que son importantes y útiles), la auto-eficacia académica (la confianza en las propias habilidades para completar las tareas asignadas) y la motivación intrínseca (el disfrute de los trabajos y actividades de aprendizaje).

La asociación preliminar establecida en la literatura entre sentido de pertenencia y motivación, también puede ser explorada a través de variables motivacionales adicionales. Una variable asociada positivamente al sentido de pertenencia - pero no como parte del factor motivación - ha sido el apoyo de los padres, aunque igualmente hay evidencia que apunta en el sentido contrario, es decir, que una vinculación fuerte a la propia familia puede significar que los alumnos no sientan la necesidad de establecer un sentido de pertenencia con su institución de estudios (Hurtado y Carter, 1997; Hurtado y Ponjuan, 2005;
Hausmann et al., 2007). También, se ha verificado la influencia del apoyo de los padres sobre la motivación de los estudiantes en la medida que ayuda, por ejemplo, a que los alumnos valoren sus metas académicas o sientan confianza en que pueden lograr los resultados académicos esperados (Ethington, 1990; Turner, Chandler y Heffer, 2009). De ahí que sea importante explorar más la asociación entre sentido de pertenencia y apoyo de los padres como parte de la motivación de los estudiantes. Asimismo, las características que tiene la enseñanza recibida por parte de los estudiantes tienen incidencia sobre su motivación (Loes y Pascarella, 2015). Si bien la relación con los profesores y sentido de pertenencia ha sido establecida (O’Keeffe, 2013), es necesario verificar la relación entre las características de la enseñanza, motivación de los estudiantes y su sentido de pertenencia. Esto es especialmente relevante en países en los que este tipo de estudios son más bien escasos, como es el caso de Chile y, en general, de Latinoamérica.

\section{Hipótesis de trabajo}

1. Siguiendo lo que se ha descrito en la literatura, esperamos que a nivel general de toda la población y a lo largo de los años de estudio de los estudiantes, los tres factores estudiados: motivación, clima organizacional e integración social se asocien positivamente al sentido de pertenencia. Esto demostrará que los resultados obtenidos en poblaciones de universitarios de otros países y que sustentan fundamentalmente los estudios sobre estas variables, pueden ser generalizados a la realidad chilena, a pesar de sus diferencias culturales.

2. No obstante, y aunque estas variables permanezcan relevantes a lo largo de los años de estudio, también esperamos que, siguiendo lo sugerido por la literatura, los niveles de asociación de los factores personales (motivación) e institucionales (integración social y clima institucional) con el sentido de pertenencia varíen en los diversos años de carrera debido a las fluctuaciones esperables 
en la valoración de los estudiantes por este tipo de variables. Específicamente, presumimos que en los primeros años de estudio la motivación sea la variable más relevante para el sentido de pertenencia de los estudiantes, en la medida que al iniciar sus carreras tienden a apoyarse en sus recursos personales, mientras que las variables integración social y clima organizacional aumenten su relevancia progresivamente en los estudiantes, puesto que van requiriendo más los apoyos institucionales en tanto avanzan en sus carreras.

3. Adicionalmente, los instrumentos diseñados para medir la motivación y la integración social han incorporado subescalas para medir de manera más precisa los aspectos específicos de estas variables. De esta manera, aunque suponemos que cada una de las subescalas sea relevante para explicar la variable sentido de pertenencia, también aguardamos diferencias en torno a su peso explicativo. Específicamente, esperamos que la motivación intrínseca y la valoración por los estudios universitarios sean más relevantes que la motivación familiar para explicar el sentido de pertenencia de los estudiantes a lo largo de los diferentes años de la carrera, optando por la perspectiva de Freeman, Anderman y Jensen (2007). Preveemos que las subescalas de integración social con los compañeros y los profesores sean los principales constructos que expliquen el sentido de pertenencia a lo largo de sus años de estudios. Si bien las actividades grupales en el aula son relevantes para el sentido de pertenencia, nosotros esperamos que los alumnos valoren más las relaciones personales que establecen no sólo dentro del aula sino también fuera de ella.

\section{Metodología Participantes}

La muestra consistió en 459 estudiantes universitarios de la carrera de Administración de una universidad chilena pública y regional que cuenta con dos campus;
335 estudiantes de la muestra pertenecen al campus 1 ubicado en la misma ciudad y región en la que se encuentra la sede central de la universidad, y 124 estudiantes al campus 2, ubicado en otra ciudad y región del país. En este sentido, se invitó a participar a todos los estudiantes de la carrera señalada, sin importar otro tipo de características individuales como edad, sexo, etcétera. De esta forma, el criterio de selección de muestra estuvo basado en la pertenencia a uno de los campus y en el estar cursando la carrera de Administración. De esta muestra, el 57.3\% son mujeres y el $42.7 \%$ son varones. Los grupos etarios mayoritarios se distribuyen de la siguiente manera: $36 \%$ de los estudiantes está entre los 20 y 21 años de edad, $25 \%$ entre los 17 y $19 ; 25 \%$ entre los 22 y 23 , y el $14 \%$ son los estudiantes mayores de 24 años.

\section{Instrumentos}

Se elaboraron cuatro instrumentos para medir a) el sentido de pertenencia, b) la integración social, c) el clima institucional y d) la motivación. En una primera etapa, un año antes de la toma definitiva de datos, estos instrumentos fueron sometidos a un juicio de expertos y a un pilotaje con 32 estudiantes, lo que permitió la conformación final de cada uno de los instrumentos. Estos instrumentos se responden con una escala Likert de cinco valores, que medía el grado de acuerdo o desacuerdo con cada uno de los ítems (con el 1 representando muy en desacuerdo; 2 en desacuerdo; 3 ni de acuerdo, ni en desacuerdo; 4 de acuerdo, y 5 muy de acuerdo). Aquellos ítems de respuesta inversa fueron invertidos para su posterior análisis siguiendo los protocolos habituales. Los detalles de los instrumentos se explican a continuación.

Sentido de pertenencia: para la elaboración de esta escala se utilizó como base el instrumento confeccionado por Bollen y Hoyle (1990). De esta forma, el instrumento se construyó a partir de cuatro ítems que medían el grado de acuerdo o desacuerdo con afirmaciones tales como Me siento parte de la universidad y Me gusta estar en esta carrera. Un posterior análisis 
factorial exploratorio con método de máxima verosimilitud respalda esta estructura de un factor con un $54.48 \%$ de la varianza explicada para el primer factor (Eigenvalues: Factor 1: 2.18, Factor 2: 0.73, Factor 3: 0.65, etcétera) que incluye las cuatro preguntas (Matriz de Componentes: 0.73, 0.75, 0.47, 0.53). En relación con la fiabilidad del instrumento, el valor a de Cronbach es de $a=0.71$, lo que se considera como aceptable.

Integración social: esta escala se construyó tomando como referencia el National Survey of Student Engagement (Kuh et al., 2008). Incluye 11 preguntas que, después de un análisis factorial exploratorio con método de máxima verosimilitud y rotación varimatrix, se agruparon en tres factores diferentes. El primer factor mide la percepción de los estudiantes sobre las actividades de aula con base en el trabajo grupal (tres preguntas, $25.94 \%$ de la varianza explicada; Eigenvalue factor 1: 3.64; Matriz de componentes: 0.91, 0.65, 0.59); el segundo se relaciona con la interacción de los estudiantes con sus compañeros (cuatro preguntas, $10.49 \%$ de la varianza explicada; Eigenvalue factor 2: 1.40; Matriz de Componentes: 0.81, 0.60, 0.43, 0.39), y el tercero se refiere a la interacción de los estudiantes con los profesores (cuatro preguntas, $6.30 \%$ de la varianza explicada; Eigenvalue factor 3: 1.18; Matriz de Componentes: 0.62, 0.58, 0.43, 0.34). Para estos factores los valores a de Cronbach fueron aceptables: a Percepción actividades en aula $=0.79$, a Interacción con compañeros $=0.69$, a Interacción con profesores $=0.61$.

Clima institucional: la escala incluye cuatro ítems adaptados del instrumento de Nuñez (2009). Una de las principales razones para adaptar los ítems radicó en eliminar el componente racial que es parte del cuestionario original. Por ejemplo, mientras el instrumento original cuenta con ítems tales como Hay mucha tensión racial en el campus y He escuchado a los profesores expresar estereotipos sobre los grupos raciales presentes en las clases, el presente instrumento incluye afirmaciones tales como Siento que las relaciones interpersonales de los estudiantes son tensas o Creo que las relaciones entre los estudiantes y los profesores son buenas. $\mathrm{El}$ análisis factorial exploratorio con método de máxima verosimilitud, arrojó una estructura de un factor con un 50.31 de la varianza (Eigenvalue factor 1: 2.01, factor 2: 0.989, etcétera. Matriz de componentes: 0.84, 0.65, 0.40, 0.37). A su vez, el valor a de Cronbach de esta escala es aceptable $\alpha=0.67$.

Motivación: para la construcción de esta escala se consideraron los instrumentos previamente diseñados por Freeman et al. (2007) y por Cabrera, Nora y Castañeda (1993). De esta forma, la escala consiste en 11 ítems que, a partir del análisis factorial exploratorio con método de máxima verosimilitud y rotación varimatrix, indicó la presencia de tres factores diferentes. Estos son claros indicadores de dimensiones de la motivación. Los primeros dos factores reflejan las principales dimensiones del instrumento de Freeman et al. (2007) con preguntas tales como Es importante para mí titularme de esta carrera; Creo que esta carrera me dará el estándar de vida que deseo; Me desmotivo cuando saco malas notas, y Creo que las materias son demasiado densas para mí. El último factor se centra en la relevancia del apoyo familiar siguiendo el instrumento de Cabrera et al. (1993) e incluye preguntas tales como: Mi familia me incentiva a ir a la universidad y Mi familia me alienta a terminar mi carrera. De este modo, los tres factores del instrumento son: a) la valoración por los estudios universitarios (cuatro preguntas, 24.25\% de la varianza explicada; Eigenvalue factor 1: 3.24; Matriz de Componentes: $0.82,0.89,0.60,0.49)$, b) la motivación intrínseca (cuatro preguntas, $14.31 \%$ de la varianza explicada; Eigenvalue factor 2: 1.96; Matriz de Componentes: $0.89,0.72,0.62,0.55)$ y c) apoyo familiar (tres preguntas, 5.98\% de la varianza; Eigenvalue factor 3: 1.35; Matriz de Componentes: 0.53, 0.44, 0.37). Los valores a de Cronbach para cada una de estas subescalas denotan un alto grado de fiabilidad en las dos primeras subescalas: a Valoración estudios universitarios $=0.79$, a motivación intrínseca $=0.80$. La tercera subescala es de $\alpha$ apoyo familiar $=0.46$. 


\section{Análisis Estadísticos}

Previo a la realización de los análisis estadísticos, se evaluó la distribución de las variables estudiadas, mismas que no resultaron normales. De esta forma, para evaluar la hipótesis 1 , se realizaron correlaciones Rho de Spearman. Para evaluar la hipótesis 2, primero se dividió la población en tres grupos temporales de acuerdo con los años de permanencia: estudiantes al inicio de la carrera que comprende los primeros tres semestres $(\mathrm{N}=158)$; estudiantes en la etapa media de su formación que comprende los semestres $4^{\circ}$ al $6^{\circ}(\mathrm{N}=160)$ y estudiantes que finalizan sus estudios entre los semestres $7^{\circ}$ al $9^{\circ}(\mathrm{N}=141)$. A continuación, se efectuaron regresiones lineales con método de introducción y por pasos en cada uno de los grupos de años de permanencia en los que se dividió la población.

Finalmente, para evaluar la hipótesis 3 , que intenta establecer separadamente el efecto de las subescalas de las variables motivación e integración social sobre el sentido de pertenencia, en primera instancia se creó una variable que controla el efecto del clima institucional sobre la variable dependiente Sentido de pertenencia. Se tomó esta decisión para establecer el valor explicativo de estas subescalas omitiendo el valor atribuible a la variable Clima institucional. Por tanto, a partir del residuo de la regresión lineal para el Clima institucional sobre el Sentido de pertenencia, se creó la variable Res-pertenencia que fue sometida separadamente a regresiones lineales múltiples con método en entrada y por pasos frente a las subescalas de las variables motivación e integración social.

\section{Resultados}

En la tabla 1 se aprecian los estadísticos descriptivos $(\mathrm{M} \pm \mathrm{DE})$ de todas las variables estudiadas en la muestra total y en la subdivisión en grupos de desarrollo académico.

En relación con la hipótesis 1, los análisis correlacionales muestran una asociación positiva de las variables estudiadas (Motivación: $\mathrm{Rho}=0.38$, $\mathrm{p}<0.01$; Integración social: Rho $=0.39, \mathrm{p}<0.01$; Clima institucional: Rho $=0.38, \mathrm{p}<0.01)$ con el sentido de pertenencia. De esta forma, para el caso de las tres variables, un aumento en cada una de ellas se asocia a un aumento en el sentido de pertenencia en la percepción de los estudiantes.

Para la hipótesis 2 se realizaron modelos de regresión lineal con método de introducción y por pasos para los tres grupos de años de permanencia en los que se dividió a la población (ver tabla 2). Los resultados entre los dos modelos de regresión muestran una gran estabilidad entre ellos al destacar que las variables que no son significativas con el método de introducción son finalmente descartadas en la regresión por pasos (véase en la tabla 2, la variable integración en el grupo inicio de la carrera y la variable motivación en el grupo de finalización de los estudios). Asimismo, el grupo de la "etapa media de formación" es claramente consistente al aplicarle los dos métodos de extracción, ya que mantiene su estructura en ambos. En los tres grupos y utilizando los dos métodos de análisis, podemos concluir que las variables explican un porcentaje relevante del fenómeno del sentido de pertenencia (ver valores $\mathrm{R} 2$ en tabla 2, alrededor de un 25\%). En este sentido, no hay que olvidar que el sentido de pertenencia se trata en sí mismo de un fenómeno altamente multifactorial. Por otro lado, todas las relaciones son de tipo positiva o directa, es decir, que un incremento de una de las variables independientes se asocia a un incremento en la variable dependiente denominada sentido de pertenencia.

Específicamente, al analizar las percepciones de los estudiantes al inicio de la carrera se puede establecer que la variable integración social no es relevante para explicar el sentido de pertenencia y la variable motivación es la que explica con más robustez el fenómeno (ver valores beta en tabla 2). Por otro lado, al analizar las percepciones de los estudiantes en etapa media de formación, al ser retenida en el modelo, la variable integración social se vuelve relevante para 
Tabla 1. Estadísticos descriptivos $(M \pm D E)$ de todas las variables estudiadas, para la población completa y las divisiones por año de desarrollo de la carrera

\begin{tabular}{|l|c|c|c|c|}
\cline { 2 - 5 } \multicolumn{1}{c|}{} & $\begin{array}{c}\text { Población Total } \\
\mathbf{N}=\mathbf{4 5 9}\end{array}$ & $\begin{array}{c}\text { Inicio de la carrera } \\
\mathbf{N}=\mathbf{1 5 8}\end{array}$ & $\begin{array}{c}\text { Etapa media de } \\
\text { formación } \\
\mathbf{N}=160\end{array}$ & $\begin{array}{c}\text { Finalización de los } \\
\text { estudios } \\
\mathbf{N}=\mathbf{1 4 1}\end{array}$ \\
\hline Pertenencia & $14.68 \pm 2.86$ & $14.84 \pm 2.95$ & $14.64 \pm 2.77$ & $14.53 \pm 2.87$ \\
\hline Clima Inst. & $14.74 \pm 2.55$ & $14.72 \pm 2.67$ & $14.53 \pm 2.44$ & $15.00 \pm 2.52$ \\
\hline Mot. Familiar & $18.04 \pm 2.37$ & $18.20 \pm 2.11$ & $18.18 \pm 2.53$ & $17.70 \pm 2.45$ \\
\hline Mot. Valoración & $17.04 \pm 2.53$ & $17.50 \pm 2.46$ & $17.09 \pm 2.58$ & $16.48 \pm 2.45$ \\
\hline Mot. Intrínseca & $9.42 \pm 2.25$ & $9.46 \pm 2.43$ & $9.18 \pm 2.25$ & $9.66 \pm 2.01$ \\
\hline Mot. Total & $44.52 \pm 2.25$ & $45.17 \pm 4.89$ & $44.45 \pm 4.79$ & $43.85 \pm 4.54$ \\
\hline I. S. Trab. Grup. & $12.50 \pm 1.88$ & $12.14 \pm 1.98$ & $12.69 \pm 1.81$ & $12.68 \pm 1.80$ \\
\hline I. S. Amigos & $13.85 \pm 3.17$ & $13.53 \pm 3.18$ & $13.96 \pm 3.23$ & $14.08 \pm 3.07$ \\
\hline I. S. Profesores & $14.24 \pm 2.57$ & $13.82 \pm 2.49$ & $14.50 \pm 2.81$ & $14.42 \pm 2.32$ \\
\hline Int. Soc. Total & $40.60 \pm 5.93$ & $39.50 \pm 5.99$ & $41.16 \pm 6.00$ & $41.19 \pm 5.66$ \\
\hline
\end{tabular}

Fuente: elaboración propia.

Tabla 2. Modelos de regresión realizados con método de introducción y por pasos para explicar el efecto de las variables independientes: motivación, integración social y clima institucional sobre la variable dependiente sentido de pertenencia en diferentes etapas de la formación universitaria

\begin{tabular}{|c|c|c|c|c|}
\hline Método & & Inicio de la carrera & Etapa media de formación & Finalización de los estudios \\
\hline \multirow{4}{*}{ 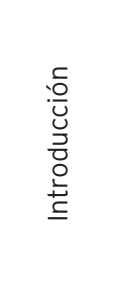 } & Valor regresión & $\mathrm{R}^{2}=.30, \mathrm{~F}_{(3)}=22.43^{* * *}$ & $\mathrm{R}^{2}=0.28, \mathrm{~F}_{(3)}=20.49 * * *$ & $\mathrm{R}^{2}=0.25, \mathrm{~F}_{(3)}=15.63^{* * *}$ \\
\hline & Motivación & Beta $=0.38 * * *$ & Beta $=0.23 * *$ & Beta $=0.13$ \\
\hline & Integración & Beta $=0.12$ & Beta $=0.29 * * *$ & Beta $=0.24^{* *}$ \\
\hline & Clima & Beta $=0.18^{*}$ & Beta $=0.19 * *$ & Beta $=0.28^{* * *}$ \\
\hline \multirow{4}{*}{ 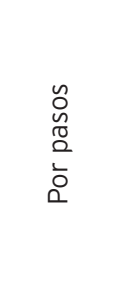 } & Valor regresión & $\mathrm{R}^{2}=0.29 \mathrm{~F}_{(2)}=31.89 * * *$ & $\mathrm{R}^{2}=0.28 \mathrm{~F}_{(3)}=20.49 * * *$ & $\mathrm{R}^{2}=0.24 \mathrm{~F}_{(2)}=22.05^{* * *}$ \\
\hline & Motivación & Beta $=0.41^{* * *}$ & Beta $=0.23 * *$ & Descartada \\
\hline & Integración & Descartada & Beta $=0.29 * * *$ & Beta $=0.29 * * *$ \\
\hline & Clima & Beta $=0.22 * *$ & Beta $=0.19 * *$ & Beta $=0.32 * * *$ \\
\hline
\end{tabular}


comprender el fenómeno del sentido de pertenencia. En esta etapa media del desarrollo académico, las tres variables independientes son relevantes y robustas para explicar el fenómeno. Finalmente, y en línea con la hipótesis 2, los estudiantes que se encuentran en etapa de finalización de sus estudios explican su sentido de pertenencia a partir de las variables integración social y clima organizacional, siendo descartada la variable motivación. En su conjunto, estos resultados entregan soporte a la hipótesis 2 ya que el sentido de pertenencia se muestra como un fenómeno dinámico en torno al peso y validez de las variables estudiadas a lo largo de la formación académica de los estudiantes universitarios y no como un fenómeno estable que se manifiesta de la misma manera a lo largo de los diferentes años de estudio.

En relación con la hipótesis 3, los análisis de las regresiones de las subescalas de la variable motivación sobre la variable Res-pertenencia (tabla 3) muestran claramente que la valoración que expresan los estudiantes por los estudios universitarios es la única variable que realmente explica de manera positiva, consistente y constante a lo largo de la carrera académica, el sentido de pertenencia. De esta forma, las variables motivación intrínseca y motivación familiar, son descartadas de todos los modelos a lo largo de la formación académica.

Por otro lado, siguiendo con la hipótesis 3, la segunda parte de ésta replicó los análisis realizados en las regresiones anteriores, pero esta vez para las subescalas de la variable integración social. En este sentido, los resultados (tabla 4) muestran que las subescalas de la variable integración social explican débilmente el sentido de pertenencia durante el inicio de la etapa académica (ver valores R2 en tabla 3). De hecho, solamente la integración con los amigos es una variable relevante en la regresión efectuada con método de análisis por pasos. En los siguientes años de formación académica, la única variable relevante para explicar el sentido de pertenencia es la integración con los profesores (ver tabla 3). Sin importar el tipo de método utilizado en la regresión, esta variable es la única que explica de manera positiva la pertenencia de acuerdo con lo expresado por los estudiantes.

\section{Tabla 3. Regresiones de las subescalas de la variable motivación sobre la variable Res-pertenencia, en diferentes etapas de la formación universitaria}

\begin{tabular}{|c|c|c|c|c|}
\hline Método & & Inicio de la carrera & Etapa media de formación & Finalización de los estudios \\
\hline \multirow{4}{*}{ 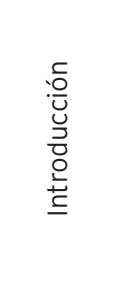 } & Valor regresión & $\mathrm{R}^{2}=0.20, \mathrm{~F}_{(3)}=12.71^{* * *}$ & $\mathrm{R}^{2}=0.14, \mathrm{~F}_{(3+)}=8.57 * * *$ & $\mathrm{R}^{2}=0.12, \mathrm{~F}_{(3)}=6.68 * * *$ \\
\hline & Valoración estudios & Beta $=0.40^{* * *}$ & Beta $=0.31 * * *$ & Beta $=0.36 * * *$ \\
\hline & Intrínseca & Beta $=0.09$ & Beta $=0.12$ & Beta $=-0.06$ \\
\hline & Familia & Beta $=-0.01$ & Beta $=0.07$ & Beta $=0.01$ \\
\hline \multirow{4}{*}{ 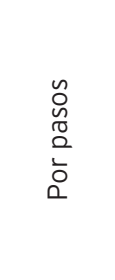 } & Valor regresión & $\mathrm{R}^{2}=0.19 \mathrm{~F}_{(1)}=36.59 * * *$ & $\mathrm{R}^{2}=0.12 \mathrm{~F}_{(1)}=21.54 * * *$ & $\mathrm{R}^{2}=0.12 \mathrm{~F}_{(1)}=19.51^{* * *}$ \\
\hline & Valoración estudios & Beta $=0.43^{* * *}$ & Beta $=0.34^{* * *}$ & Beta $=0.35^{* * *}$ \\
\hline & Intrínseca & Descartada & Descartada & Descartada \\
\hline & Familiar & Descartada & Descartada & Descartada \\
\hline
\end{tabular}

Fuente: elaboración propia.

Nota: $+p<0.06, * p<0.05, * * p<0.01, * * * p<0.001$ 
Tabla 4. Regresiones de las subescalas de la variable integración social sobre la variable Res-pertenencia, en diferentes etapas de la formación universitaria

\begin{tabular}{|c|c|c|c|c|}
\hline Método & & Inicio de la carrera & Etapa media de formación & Finalización de los estudios \\
\hline \multirow{4}{*}{ 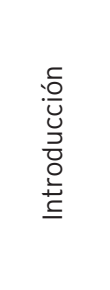 } & Valor regresión & $R^{2}=0.03, F_{(3)}=1.91$ & $\mathrm{R}^{2}=0.18, \mathrm{~F}_{(3)}=11.25^{* * *}$ & $\mathrm{R}^{2}=0.10, \mathrm{~F}_{(3)}=5.29 * * *$ \\
\hline & Trabajo Grupo & Beta $=0.00$ & Beta $=0.05$ & Beta $=0.01$ \\
\hline & Int. amigos & Beta $=0.12$ & Beta $=0.09$ & Beta $=0.10$ \\
\hline & Int. Profesores & Beta $=0.10$ & Beta $=0.355^{* * *}$ & Beta $=0.25^{*}$ \\
\hline \multirow{4}{*}{ 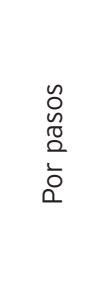 } & Valor regresión & $\mathrm{R}^{2}=0.12 \mathrm{~F}_{(1)}=4.32 *$ & $\mathrm{R}^{2}=0.16 \mathrm{~F}_{(1)}=31.02 * * *$ & $\mathrm{R}^{2}=0.09 \mathrm{~F}_{(1)}=14.52 * * *$ \\
\hline & Trabajo Grupo & Descartada & Descartada & Descartada \\
\hline & Int. amigos & $0.16 *$ & Descartada & Descartada \\
\hline & Int. Profesores & Descartada & Beta $=0.40 * * *$ & Beta $=0.30 * * *$ \\
\hline
\end{tabular}

Fuente: elaboración propia.

Nota: $+p<0.06,{ }^{*} p<0.05,{ }^{* *} p<0.01, * * * p<0.001$.

\section{Discusión y comentarios finales}

Dada la importancia del sentido de pertenencia para los estudios superiores, la presente investigación tuvo como objetivo explorar este fenómeno y su asociación con diversas variables personales e institucionales en estudiantes chilenos. De los múltiples factores que derivan de modelos acerca de la permanencia o deserción de los estudiantes de sus estudios superiores, se individualizaron tres que se relacionan con el sentido de pertenencia: la motivación, la integración social y el clima organizacional (Hurtado y Carter, 1997; Strayhon, 2008; Freeman, Anderman y Jensen, 2007). La presente investigación reafirma que, desde la perspectiva de los estudiantes participantes, estos factores se relacionan positivamente con el sentido de pertenencia que, por tanto, puede ser conceptualizado como un factor aglutinador que permite dar una mirada panorámica respecto a las variables que inciden en que los estudiantes desarrollen exitosamente sus estudios universitarios.
Esto constituye un aporte a la literatura en la medida que el sentido de pertenencia suele ser incorporado en las investigaciones como una variable más entre otras impidiendo una mirada más focalizada e integral de esta experiencia considerada relevante para la permanencia de los estudiantes en la universidad (Nora y Cabrera, 1993).

Asimismo, aunque basados en instrumentos existentes, este trabajo contribuye con nuevos instrumentos auto-elaborados para dar cuenta de su objetivo de investigación y suplir la ausencia relativa de instrumentos validados para la población chilena y para la especificidad del estudio. No obstante, aunque estos instrumentos fueron robustos en términos de su consistencia y fiabilidad, es necesario que estudios futuros los repliquen en diferentes poblaciones universitarias, en distintos centros de educación superior y que incluyan una alta gama de programas de estudio. Igualmente, al centrarse este trabajo en un aspecto altamente multifactorial y subjetivo, 
como lo es el sentido de pertenencia, futuros estudios deberían integrar aproximaciones cualitativas que permitan seguir integrando con mayor profundidad la perspectiva de los estudiantes y complementando los análisis más institucionales existentes en la literatura sobre el mejoramiento de la educación superior y el desarrollo formativo de los estudiantes (Donoso, Donoso y Arias, 2010; Gallifa y Batallé, 2010).

Específicamente, los resultados de esta investigación permiten corroborar la hipótesis 1 y, consecuentemente, confirmar que, aunque el sentido de pertenencia constituye una experiencia subjetiva, su correlación positiva con los tres factores ya señalados permiten conceptualizarla en referencia a ámbitos sociales que no están restringidos a la intimidad de los estudiantes. Así, el sentido de pertenencia, tal como ha sido analizado en este texto considerando las percepciones de los propios estudiantes, vincula las experiencias de éstos con factores personales referidos a su contexto afectivo y social más próximo, así como factores institucionales que dan cuenta de su interacción con actores relevantes del contexto académico en el cual se desarrollan. El constructo teórico resultante posibilita no sólo una mejor comprensión de por qué los estudiantes deciden permanecer en sus programas de estudios al sentirse parte de las instituciones que los imparten, sino que también sugiere la importancia de los contextos en los cuales los estudiantes desarrollan su vida académica y el cómo contribuyen a que completen con éxito sus estudios (Haussmann et al., 2007). Particularmente, el estudio ofrece una mirada desde la realidad chilena, y por tanto latinoamericana, más bien ausente en la literatura y que es complementaria a la desarrollada en los estudios sobre sentido de pertenencia que han sido realizados mayormente en contextos anglosajones.

De este modo, la corroboración de la hipótesis 2 ofrece un análisis más detenido sobre el comportamiento de estas variables en la percepción de los estudiantes chilenos a lo largo de sus años de estudio.
Si bien todas las variables son importantes, la variable motivación influye más decisivamente en que los estudiantes en la primera parte de sus estudios se sientan parte de la institución, mientras que en la etapa media las tres variables de motivación, integración social, y clima institucional son importantes. Finalmente, para la última etapa, los estudiantes consideran las variables de integración social y clima organizacional como las más significativas. Estos hallazgos aportan una mirada longitudinal de acuerdo con la cual los estudiantes inicialmente recurrirían fundamentalmente a sus propios recursos para luego ir incorporando crecientemente dentro de su bagaje los recursos institucionales con los que interactúa; proceso que les ayuda a 'sentirse parte' y finalizar exitosamente sus estudios. Esta constatación está algo ausente en las investigaciones sobre sentido de pertenencia al estar más bien centradas en los primeros años de estudios (Hoffman et al., 2002/2003). Desde la perspectiva de las instituciones universitarias, dicho enfoque implica la importancia de promover los factores personales con que los estudiantes ingresan a sus estudios superiores y sobre los cuales construyen crecientemente su relación con las normas y prácticas institucionales que son requeridas para su formación académica (Tinto, 2012).

No obstante, la corroboración de la hipótesis 3 complementa el análisis anterior y sugiere algunas matizaciones importantes a las implicancias que emergen del estudio. De acuerdo con los resultados obtenidos a partir de los estudiantes, en la variable motivación la subescala valoración por los estudios universitarios es significativa en comparación a las otras dos subescalas de motivación intrínseca y apoyo familiar. En relación con la variable de integración social, los estudiantes en una primera etapa señalan a la subescala interacción con sus compañeros como la más significativa por sobre las actividades cooperativas en el aula y la interacción con los profesores. Sin embargo, en los grupos con un mayor número de años en el programa de estudio, la variable sobre 
interacción con los profesores pasa a ser la significativa, mientras las otras variables no lo son. Estos hallazgos, por tanto, suscitan interrogantes respecto a cómo los estudiantes estarían valorizando sus experiencias y la pertinencia que éstas tienen con las relaciones de pertenencia que procuran desarrollar con sus instituciones educativas. Asimismo, la creciente valoración por la relación con sus profesores por sobre la relación con sus pares, igualmente abre preguntas respecto a las características que adquiere el sentido de pertenencia de los estudiantes y de qué modo podría estar afectando las expectativas de éxito que buscan a través de sus estudios.

Consecuentemente, estos resultados podrían tener particular relevancia en el actual contexto en el cual los estudiantes están desarrollando sus estudios superiores y que está marcado por procesos de acreditación de las universidades chilenas que buscan certificar si éstas cuentan o no con los mecanismos institucionales para desarrollar sus proyectos educativos y formar capital humano productivo para la sociedad global (Cancino y Schmal, 2014; Fleet, Pedraja-Rejas y Rodriguez-Ponce, 2014; Espinoza y González, 2012; Rodríguez-Ponce, 2009). Si bien estos procesos toman en cuenta el parecer de los estudiantes a través de diversos instrumentos de evaluación - les hacen "sentirse parte"-, su fin podría no estar en función de las necesidades de los alumnos sino de la inserción de las universidades en los mercados nacionales e internacionales (Blackmore, 2009). En consecuencia, es posible que dicho contexto tenga como efecto que los estudiantes se vinculen con sus universidades más por el prestigio y las oportunidades laborales que les podrían reportar que por el valor intrínseco que procede de sus estudios. Asimismo, la creciente importancia que dan a su relación con los profesores podría develar el carácter pragmático de las relaciones que los estudiantes establecen para avanzar en sus estudios, mientras otras relaciones más importantes en lo afectivo con familiares y amigos son consideradas menos relevantes para su formación profesional.

Desde esa perspectiva, el presente estudio abre la necesidad, a la luz de las múltiples variables que intervienen, de seguir profundizando respecto a cómo los factores institucionales influyen en los modos a través de los cuales los estudiantes discriminan entre sus experiencias para establecer un sentido de pertenencia con sus programas de estudios. Específicamente, los resultados de esta investigación invitan a poner más atención en los actuales procesos de acreditación y en cómo podrían estar influyendo sobre las necesidades de los estudiantes y, por tanto, en el sentido de pertenencia que desarrollan. Si bien este sentido de pertenencia les podría estar ayudando a completar sus estudios, también podría estar limitando las interacciones que establecen a lo largo de sus años de estudio restringiendo igualmente su comprensión respecto a qué significaría formarse exitosamente para su futura vida profesional (Johnson y Deem, 2003; Cardoso, Carvalho y Santiago, 2011). 


\section{Referencias}

Abello, Rubén, Alejandro Díaz, María Pérez, Leandro Almeida, Irma Lagos, Jaime González y Brandee Strickland (2012), "Vivencias e implicación académica en estudiantes universitarios: adaptación y validación de escalas para su evaluación", en Estudios Pedagógicos, vol. XXXVIII, núm. 2, pp. 7-19.

Anderman, Lynley (2003), "Academic and social perceptions as predictors of change in middle school students' sense of school belonging", en The fournal of Experimental Education, vol. 72, núm. 1, pp. 5-22.

Bean, John y Barbara Metzner (1985), "A conceptual model of nontraditional undergraduate student attrition" en Review of Educational Research, vol. 55, núm. 4, pp. 485-540.

Blackmore, Jill (2009), "Academic pedagogies, quality logics and performative universities: evaluating teaching and what students want", en Studies in Higher Education, vol. 34, núm. 8, pp. 857-872.

Bollen, Kenneth y Rick Hoyle (1990), "Perceived cohesion: a conceptual and empirical examination", en Social Forces, vol. 69, núm. 2, pp. 479-504.

Braxton, John y Shederick McClendon (2001/2002), "The fostering of social integration and retention through institutional practice", en fournal College Student Retention, vol. 3, núm. 1, pp. 57-71.

Brunner, José Joaquín (2013), The rationale for higher education investment in Ibero-America, París, OECD Development Centre [Working paper núm. 319].

Cabrera, Alberto, Amaury Nora y María Castañeda (1993), "College persistence. Structural equations modeling test of an integrated model of student retention", en Journal of Higher Education, vol. 64, núm. 2, pp. 123-139.

Canales, Andrea y Danae de los Ríos (2009), "Retención de estudiantes vulnerables en la educación universitaria chilena”, en Calidad en la Educación, núm. 30, pp. 49-89.

Cancino, Víctor y Rodolfo Schmal (2014), "Sistema de acreditación universitaria en Chile: ¿cuántos hemos avanzado?", en Estudios Pedagógicos, vol. XI, núm. 1, pp. 41-60.
Cardoso, Sónia, Teresa Carvhalo y Rui Santiago (2011), "From students to consumers: reflections on the marketization of Portuguese higher education", en European fournal of Education, vol. 46, núm. 2, pp. 271284.

Castaño, Elkin, Santiago Gallón, Karoll Gómez y Johanna Vásquez (2006), "Análisis de los factores asociados a la deserción y graduación estudiantil universitaria", en Lecturas de Economía, núm. 65, pp. 9-36.

De Vries, Wietse, Patricia León, José Romero, Ignacio Hernández (2011), “¿Desertores o decepcionados? Distintas causas para abandonar los estudios universitarios", en Revista de la Educación Superior, vol. XL, núm. 160, pp. 29-49.

Díaz, Christian (2008), "Modelo conceptual para la deserción estudiantil universitaria chilena", en Estudios Pedagógicos, vol. XXXIV, núm. 2, pp. 65-86.

Donoso, Sebastián y Ernesto Schiefelbein (2007), "Análisis de los modelos explicativos de retención de estudiantes en la universidad: una visión desde la desigualdad social", en Estudios Pedagógicos, vol. XXXIII, núm. 1, pp. 7-27.

Donoso, Sebastián, Gonzalo Donoso y Oscar Arias (2010), "Iniciativas de retención de estudiantes en educación superior", en Calidad en la Educación, núm. 33, pp. 16-61.

Eaton, Shevawn y John Bean (1995), "An approach/ avoidance behavioral model of college student attrition", en Research in Higher Education, vol. 36, núm. 6, pp. 617-645.

Espinoza, O. y Luis Eduardo González (2012), "Estado actual del sistema de aseguramiento de la calidad y el régimen de acreditación en la educación superior en Chile", en Revista de la Educación Superior, vol. XLI, núm. 162, pp. 87-109.

Ethington, Corrina (1990), "A psychological model of student persistence", en Research in Higher Education, vol. 31, núm.3, pp. 279-283.

Fleet, Nicolas, Liliana Pedraja-Rejas, y Emilio RodriguezPonce (2014), "Acreditación institucional y factores de 
la calidad universitaria en Chile", en Interciencia, vol. 39, núm. 7, pp. 450-457.

Freeman, Tierra, Lynley Anderman, y Jane Jensen (2007), "Sense of belonging in college freshmen at the classroom and campus levels" en The Gournal of Experimental Education, vol. 75, núm. 3, pp. 203-220.

Gallifa, Josep y Pere Batallé (2010), "Student perceptions of service quality in a multi-campus higher education system in Spain", en Quality Assurance in Education, vol. 18, núm. 2, pp. 156-170.

Goodenow, Carol (1993), "Classroom belonging among early adolescent students: relationships to motivation and achievement", en The Fournal of Early Adolescence, vol. 13, núm. 1, pp. 21-43.

Haussmann, Leslie, Janet Schofield y Rochelle Woods (2007), "Sense of belonging as a predictor of intentions to persist among African American and white first-year college students", en Research in Higher Education, vol. 48, núm. 7, pp. 803-839.

Himmel, Erika (2002), "Modelos de análisis de la deserción estudiantil en la educación superior", en Revista Calidad de la Educación Superior, vol. 17, núm. 2, pp. 91-108.

Hoffman, Marybeth, Jayne Richmond, Jennifer Morrow y Kandice Salomone (2002/2003), "Investigating 'sense of belonging' in first-year college students", en fournal College Student Retention, vol. 4, núm. 3, pp. 227256.

$\mathrm{Hu}$, Shouping (2011), "Reconsidering the relationship between student engagement and persistence in college", en Innovative Higher Education, vol. 36, pp. 97 106.

Hurtado, Sylvia y Deborah Carter (1997), "Effects of college transition and perceptions of the campus racial climate on Latino college students' sense of belonging", en Sociology of Education, vol. 70, núm. 4, pp. 324-345.

Hurtado, Sylvia y Luis Ponjuan (2005), "Latino educational outcomes and the campus climate", en Journal of Hispanic Higher Education, vol. 4, núm. 3, pp. 235-251.
Johnson, Rachel y Rosemary Deem (2003), "Talking of students: tensions and contradictions for the manageracademic and the university in contemporary higher education", en Higher Education, vol. 46, pp. 289-314.

Johnson, Dawn, Matthew Soldner, Jeannie Leonard, Patty Alvarez, Karen Inkelas, Heather Rowan-Kenyon y Susan Longerbeam (2007), "Examining sense of belonging among first-year undergraduates from different racial/ethnic groups", en Fournal of College Student Development, vol. 48, núm. 5, pp. 525-542.

Kember, David, Kenneth Lee y Natalia Li (2001), "Cultivating a sense of belonging in part-time students", en International Fournal of Lifelong Education, vol. 20, núm. 4, pp. 326-341.

Kember, David y Doris Leung (2004), "Relationship between the employment of coping mechanisms and a sense of belonging for part-time students", en Educational Psychology: an International fournal of Experimental Educational Psychology, vol. 24, núm. 3, pp. 345-357.

Kuh, George, Ty Cruce, Rick Shoup, Jilian Kinzie, y Robert Gonyea (2008), "Unmasking the effects of student engagement on first-year college grades and persistence", en The fournal of Higher Education, vol. 79, núm. 5, pp. 540-563.

Locks, Angela, Sylvia Hurtado, Nicholas Bowman, y Leticia Oseguera (2008), "Extending notions of campus climate and diversity to students' transition to college", en The Review of Higher Education, vol. 31, núm. 3, pp. 257-285.

Loes, Chad y Ernest Pascarella (2015), "The benefits of good teaching extend beyond course achievement", en fournal of the Scholarship of Teaching and Learning, vol. 15, núm. 2, pp. 1-13.

Maestas, Ricardo, Gloria Vaquera y Linda Muñoz (2007), "Factors impacting sense of belonging at a hispanicserving institution", en Journal of Hispanic Higher Education, vol. 6, núm. 3, pp. 237-256.

Meeuwisse, Marieke, Sabine Severiens y Marise Born 
(2010), "Learning environment, interaction, sense of belonging and study success in ethnically diverse student", en Research in Higher Education, vol. 51, pp. 528-545.

Neuville, Sandrine, Mariane Frenay, Julia Schmitz, Gentiane Boudrenghien, Bernadette Noël y Vincent Wertz (2007), “Tinto's theoretical perspective and expectancy-value paradigm: a confrontation to explain freshmen's academic achievement", en Psychologica Belgica, vol. 47, núm. 1/2, pp. 31-50.

Nora, Amaury (2004), "The role of habitus and cultural capital in choosing a college, transitioning from high school to higher education, and persisting in college among minority and non-minority students", en fournal of Hispanic Higher Education, vol. 3, núm. 2, pp. 180-280.

Nora, Amaury y Alberto Cabrera (1996), "The role of perceptions of prejudice and discrimination on the adjustment of minority students to college", en fournal of Higher Education, vol. 67, núm. 2, pp. 119-148.

Nora, Amaury y Alberto Cabrera (1993), "The construct validity of institutional commitment: a confirmatory factor analysis", en Research in Higher Education, vol. 34, núm. 2, pp. 243-262.

Nuñez, Anne-Marie (2009), "A critical paradox? Predictors of Latino students' sense of belonging in college", en Journal of Diversity in Higher Education, vol. 2, núm. 1, pp. 46-61.

O'Keeffe, Patrick (2013), "A sense of belonging: improving student retention", en College Student fournal, vol. 47, núm. 4, pp. 605-613.

Osorio, Ana María, Catalina Bolancé y Maribel Castillo-Caicedo (2012), "Deserción y graduación estudiantil universitaria: una aplicación de los modelos de supervivencia”, en Revista Iberoamericana de Educación Superior, vol. 6, núm. 3, pp. 31-57.

Ostrove, Joan y Susan Long (2007), "Social class and belonging: implications for college adjustment", en The Review of Higher Education, vol. 30, núm. 4, pp. 363-389.

Read, Barbara, Louise Archer y Carole Leathwood (2003), "Challenging cultures? Student conceptions of 'belonging' and ‘isolation' at a post-1992 University”, en
Studies in Higher Education, vol. 28, núm. 3, pp. 261-277.

Rodríguez-Ponce, Emilio (2009), "El rol de las universidades en la sociedad del conocimiento y en la era de la globalización: evidencia desde Chile", en Interciencia, vol. 34, núm. 11, pp. 822-829.

Saldaña, Magdalena y Omar Barriga (2010), "Adaptación del modelo de deserción universitaria de Tinto a la Universidad Católica de la Santísima Concepción, Chile”, en Revista de Ciencias Sociales, vol. XVI, núm. 4, pp. 616-628.

Spady, William (1971), "Dropouts from higher Education: Toward an empirical model", en Interchange, vol. 2, pp. 38-62.

Strayhon, Terrell (2008), "Sentido de pertenencia. A hierarchical analysis predicting sense of belonging among Latino college students", en fournal of Hispanic Higher Education, vol. 7, núm. 4, pp. 301-320.

Tinto, Vincent (2012), Completing college; rethinking institutional action, Chicago, The University of Chicago Press.

Tinto, Vincent (2006/2007), "Research and practice of student retention: what next?", en fournal College Student Retention, vol. 8, núm. 1, pp. 1-19.

Tinto, Vincent (1993), Leaving college: rethinking the causes and cures of student attrition, Chicago, The University of Chicago Press.

Tinto, Vincent (1975), "Dropouts from higher education: a theoretical synthesis of recent research", en Review of Educational Research, vol. 45, pp. 89-125.

Turner, Erlanger, Megan Chandler y Robert Heffer (2009), "The influence of parenting styles, achievement motivation, and self efficacy on academic performance in college students", en Fournal of College Student Development, vol. 50, núm. 3, pp. 337-346.

Wilcox, Paula, Sandra Winn, Marylynn Fyvie-Gauld, (2005), "'It was nothing to do with the university, it was just the people': the role of social support in the first-year experience of higher education", en Studies in Higher Education, vol. 30, núm. 6, pp. 707-722.

Wolf-Wendel, Lisa, Kelly Ward y Jillian Kinzie (2009), "A tangled web of terms: the overlap and unique 
contribution of involvement, engagement, and integration to understanding college student success", en Journal of College Student Development, vol. 50, núm. 4, pp. 407-428.
Zepke, Nick, Linda Leach y Tom Prebble (2006), "Being learner centred: one way to improve student retention?", en Studies in Higher Education, vol. 31, núm. 5, pp. 587-600.

\section{Cómo citar este artículo:}

Pino-Vera, Teresa, Eduardo Cavieres-Fernández y José-Antonio Muñoz-Reyes (2018), “Los factores personales e institucionales en el sentido de pertenencia de estudiantes chilenos a lo largo de sus estudios superiores", en Revista Iberoamericana de Educación Superior (RIES), México, UNAM-IISUE/Universia, vol. IX, núm. 25, pp. 24-41, http://dx.doi.org/10.22201/iisue.20072872e.2019.25.337 [consulta: fecha de última consulta]. 